\title{
Parafree Center-by-Metabelian Lie Algebras
}

\author{
Zehra Velioğlu*
}

\begin{abstract}
Let $L$ be a Lie algebra. Denote the second term of the derived series of $L$ by $L^{\prime \prime}$. We define the parafree centre-by-metabelian Lie algebras. We prove that if $L$ is a parafree centre-by-metabelian, then the center of $L$ is $L^{\prime \prime}$. Moreover we show that the algebra $L / L^{\prime \prime}$ is parafree metabelian Lie algebra.

Keywords: Free Lie algebra; Parafree Lie algebra; Center-by-Metabelian.

AMS Subject Classification (2020): Primary: 17B99; Secondary: $17 B 01$.

*Corresponding author
\end{abstract}

\section{Introduction}

H. Neumann asked whether a group with the same lower central sequence as a free group is itself free or not. This question led to the definition of parafree groups. The concept of parafree groups was introduced by G. Baumslag in 1967 [1], answering H. Neumann's question in the negative. G. Baumslag has obtained some interesting results about parafree groups [2],[3]. Baumslag and his friends have taken his result on one relator groups to formulate several of these questions for one-relator parafree groups [4], [5]. Because of the close relationship between groups and Lie algebras, one would expect that parafree Lie algebras enjoy properties that are analogous to those of parafree groups. In [6] and [7], H. Baur has translated the formal arguments used for parafree groups to parafree Lie algebras and he has proved the existence of these Lie algebras. He obtained basic results which provide a solid understanding of the structure of parafree Lie algebras. In [9], N. Ekici and Z. Velioğlu considered unions of parafree Lie algebras and they prove that such unions are again parafree under some conditions. In [10], N. Ekici and Z. Velioğlu proved that the direct limit of any directed system of parafree Lie algebras exists in the variety of parafree Lie algebras. Baumslag's and Baur's works have given a start for studies in the theory of parafree Lie algebras. Although there are some works about parafree Lie algebras in the literature and many questions about them have remained unanswered. This fact and paucity of studies about parafree Lie algebras motivated us for working on these algebras. In this work we discuss parafree center-by-metabelian Lie algebras. We prove that the center of a parafree center-by-metabelian Lie algebra is its second derived algebra and the quotient algebra of a center-by-metabelian Lie algebra by its second derived algebra is parafree metabelian.

\section{Preliminaries}

Let $K$ be a field of characteristic zero and $L$ be a Lie algebra over $K$. The lower central series of $L$

$$
L=\gamma_{1}(L) \supseteq \gamma_{2}(L) \supseteq \cdots \supseteq \gamma_{n}(L) \supseteq \cdots
$$

is defined inductively by $\gamma_{2}(L)=[L, L], \gamma_{n+1}(L)=\left[\gamma_{n}(L), L\right], n \geq 2$. If $n$ is the smallest integer satisfying $\gamma_{n}(L)=0$, then $L$ is called nilpotent of class $n$.

Let $n_{1}, n_{2}, \ldots, n_{k}$ be a sequence of positive integers with $n_{i} \geq 1$ for $i=1,2, \cdots, k$. We define the polycentral series of $L$, relative to this sequence, inductively by

$$
L_{n_{1}, n_{2}, \ldots, n_{i}}=\gamma_{n_{i}}\left(\gamma_{n_{i-1}}\left(\cdots\left(\gamma_{n_{1}}(L)\right) \cdots\right)\right)
$$

Received : 04-06-2020, Accepted : 29-08-2020 
for $k \geq i$. In case $n_{1}=n_{2}=\ldots=n_{i}=2$ we write $\delta^{i}(L)=L_{n_{1}, n_{2}, \ldots, n_{i}}$ and call it the $\mathrm{i}$-th term of the derived series of $L$. If $m$ is the smallest integer satisfying $\delta^{m}(L)=\{0\}$, then $L$ is called solvable of class $m$.

We use the common abbreviation $L^{\prime}$ and $L^{\prime \prime}$ for $\gamma_{2}(L)$ and for $\delta^{2}(L)$ respectively. Then $L$ is called abelian (metabelian), if $L^{\prime}=0$ (resp. $\left.L^{\prime \prime}=0\right)$.

Definition 1. Let $L$ be a Lie algebra. The center of $L$ is denoted by $Z(L)$ and defined as

$$
Z(L)=\{z \in L:[x, z]=0 \text { for all } x \in L\} .
$$

Definition 2. If the factor Lie algebra $L / Z(L)$ is metabelian, it is said that $L$ is centre-by-metabelian. An equivalent description is to say that the second derived algebra of $L$ is contained in its center; i.e., if $\left[L^{\prime \prime}, L\right]=0$.

Definition 3. The Lie algebra $L$ is called residually nilpotent if

$$
\bigcap_{n=1}^{\infty} \gamma_{n}(L)=0
$$

Equivalently, given any non-trivial element $u \in L$ there exists an ideal $J$ of $L$ such that $u \notin J$ with $L / J$ nilpotent.

We associate the lower central series of $L$ with its lower central sequence:

$$
L / \gamma_{2}(L), L / \gamma_{3}(L), \cdots
$$

We say that two Lie algebras $L$ and $H$ have the same lower central sequence if

$$
L / \gamma_{n}(L) \cong H / \gamma_{n}(H)
$$

for every $n \geq 1$.

Definition 4. The Lie algebra $L$ is called parafree over a set $X$ if,

i) $L$ is residually nilpotent, and

ii) L has the same lower central sequence as a free Lie algebra generated by the set $X$.

The cardinality of $X$ is called the rank of $L$.

Definition 5. Let $Y$ be a subset of a parafree Lie algebra $L$. If $Y$ freely generates $L$ modulo $\gamma_{2}(L)$, then it is called paragenerating set of $L$.

We denote by $\Delta_{w}(L)$ the intersection of ideals $J$ of the Lie algebra $L$ such that $L / J$ is nilpotent.

\section{Parafree Centre-by-Metabelian Lie algebras}

In this section we consider all Lie algebras over a field of characteristic zero. Let $P$ be a parafree Lie algebra, then by the definition $P /\left[P^{\prime \prime}, P\right]$ is a center-by-metabelian Lie algebra. Since the quotient algebra of a parafree Lie algebra is parafree [11], then $P /\left[P^{\prime \prime}, P\right]$ is also parafree. One can call the algebra $P /\left[P^{\prime \prime}, P\right]$ as parafree center-by-metabelian Lie algebra.

Theorem 1. Let $P$ be a parafree Lie algebra with paragenerating set $X$. Then $X$ generates a free Lie algebra.

Proof. The proof can be found in [11].

The following Lemma is well known (see [8]).

Lemma 1. Suppose that a set $Y$ freely generates free Lie algebra $F$ modulo $\gamma_{2}(F)$. Then $Y$ freely generates $F$ modulo $\gamma_{n}(F)$, for $n=3,4, \ldots$.

Lemma 2. If a non-empty set $X$ freely generates a parafree Lie algebra $T$ modulo $\gamma_{2}(T)$, then it freely generates $T$ modulo $\gamma_{n}(T)$, where $n=3,4, \ldots$ 
Proof. Let $T$ be a parafree Lie algebra freely generated by a nonempty set $X$ modulo $\gamma_{2}(T)$. Consider $T / \gamma_{2}(T)$ :

$$
\begin{aligned}
& T / \gamma_{2}(T) \cong\left(T / \gamma_{n}(T)\right) /\left(\gamma_{2}(T) / \gamma_{n}(T)\right) \\
& =\left(T / \gamma_{n}(T)\right) /\left(\left(\gamma_{2}(T)+\gamma_{n}(T)\right) / \gamma_{n}(T)\right) \\
& =\left(T / \gamma_{n}(T)\right) / \gamma_{2}\left(T / \gamma_{n}(T)\right)
\end{aligned}
$$

By hypothesis, $X$ generates $T$ modulo $T / \gamma_{2}(T)$, then $X$ freely generates $T / \gamma_{n}(T)$ modulo its derived algebra. So by Lemma $1, X$ freely generates $T / \gamma_{n}(T)$.

Lemma 3. If $P$ is a parafree center-by-metabelian Lie algebra with paragenerating set $X$, then the left-normed basic commutators of length $\leq n$ in $X$ are independent.

Proof. Let $P$ be a center-by-metabelian Lie algebra with paragenerating set $X=\left\{x_{1}, x_{2}, \ldots\right\}$ and $F$ be the free Lie algebra on a set $Y$, where $|X|=|Y|$. So by Theorem 1, X generates a free center-by-metabelian Lie algebra. Call it as $H$. So $H=F /\left[F^{\prime \prime}, F\right]$.

Since

$$
H / H^{\prime \prime} \cong F / F^{\prime \prime},
$$

then $H / H^{\prime \prime}$ is a free metabelian Lie algebra. Let $\bar{a}_{i}=a_{i}+H^{\prime \prime}$ be the basic commutators in $H / H^{\prime \prime}$, where $a_{i} \in H_{i}$. Suppose that there exists a sum

$$
\alpha_{1} a_{1}+\ldots+\alpha_{n} a_{n}=0
$$

in $H$, where $\alpha_{i}$ are elements of the universal enveloping algebra $U\left(F / F^{\prime \prime}\right)$ of $F / F^{\prime \prime}$. Then

$$
\begin{aligned}
& \overline{\alpha_{1} a_{1}+\ldots+\alpha_{n} a_{n}}=\overline{0}, \\
& \alpha_{1} \bar{a}_{1}+\ldots+\alpha_{n} \bar{a}_{n}=0 .
\end{aligned}
$$

It is known that the left-normed basic commutators of free metabelian Lie algebras are independent. Hence

$$
\alpha_{1}=\ldots=\alpha_{n}=0
$$

and elements $a_{i}$ are independent in $H$.

Consider a parafree center-by-metabelian Lie algebra $L$ with paragenerating set $X$. The proof of Theorem 2 follows from the following lemmas.

Theorem 2. Let $L$ be a parafree center-by-metabelian Lie algebra. Then $\Delta_{w}\left(L / L^{\prime \prime}\right)=\{0\}$.

Lemma 4. The center of a parafree center-by-metabelian Lie algebra $L$ is its second derived algebra $L^{\prime \prime}$.

Proof. In order to prove the Lemma 4, we must show that $Z(L)$ is a subset of $L^{\prime \prime}$. By Lemma 3, the left-normed basic commutators of $L$ in $X$ are independent. Even though $X$ is not a generating set for $L$, every element $p \in L$ can be written modulo $\gamma_{n+1}(L)$ as a sum of basic commutators in $X$, since $X$ generates $L / \gamma_{n+1}(L)$, for $n=1,2,3, \ldots$ (Lemma 2). Hence, modulo $\gamma_{n+1}(L)$, every element $p \in L$ can be written in the form

$$
g_{1}+g_{2}+\ldots+g_{n}+h
$$

where $g_{i}$ is a sum(possibly empty) of left-normed basic commutators in $X$ of weight $i$, for $i=1,2, \ldots, n$ and $h$ is a sum(possibly empty) of basic commutators in $X$ that are in $L^{\prime \prime}$.

Since $L$ is residually nilpotent it can be embedded as a sub direct sum in the infinite direct sum $\sum_{n=2}^{\infty} L / \gamma_{n}(L)$ (Definition 4); that is, for $x \in L$,

$$
\begin{aligned}
& \phi: \\
& \quad=\left(\overline{0}, \overline{0}, \ldots, \overline{0}, \frac{(L), x+}{g_{l}+h_{l}}, \frac{\gamma_{3}(L), \ldots, x+\gamma_{n}}{g_{l}+g_{l+1}+h_{l+1}}, \ldots\right)
\end{aligned}
$$


where $l$ is the minimum integer such that $x \notin \gamma_{l+1}(L), g_{i}$ is a sum of left normed basic commutators of weight $i$ in $X$ and $h_{i}$ is a sum of central basic commutators in $X$.

Note: if $g_{i}=0$ for all $i$, then $x \in L^{\prime \prime}$.

Suppose $c \in Z(L)$ and consider $\phi(c)$. Assume that $g_{i} \neq 0$ for some $i$ and let $j$ be the minimum such $i$. Then $g_{j}$ is a non-empty sum of left-normed basis commutators all of whose weights are exactly $j$.

Then

$$
\phi(c)=\left(\overline{0}, \ldots, \overline{0}, \bar{h}_{l}, \bar{h}_{l+1}, \ldots, \overline{g_{j}+h_{j}}, \overline{g_{j}+g_{j+1}+h_{j+1}}, \ldots\right) .
$$

Let $x \in X$. Consider the image of $[c, x]$ under $\phi$.

$$
\begin{aligned}
& 0=[c, x] \\
& \phi(0)=\phi([c, x]) \\
&(\overline{0}, \overline{0}, \ldots)=\left(\overline{0}, \overline{0}, \ldots, \overline{\left[h_{l}, x\right]}, \overline{\left[h_{l+1}, x\right]}, \ldots, \overline{\left[g_{j}+h_{j}, x\right]}, \overline{\left[g_{j}+g_{j+1}+h_{j+1}, x\right]}, \ldots\right) .
\end{aligned}
$$

Since $h_{n}$ is central for all $n=1,2,3, \ldots$, we have

$$
(\overline{0}, \ldots, \overline{0}, \ldots)=\left(\overline{0}, \overline{0}, \ldots, \overline{\left[g_{j}, x\right]}, \overline{\left[g_{j}+g_{j+1}, x\right]}, \ldots\right) .
$$

Observe that $\left[g_{j}, x\right] \in \gamma_{j+1}(L)$, so $\overline{\left[g_{j}, x\right]}=\overline{0}$ in the $j$-th entry.

Now the $(j+1)$-th entry is $\overline{\left[g_{j}+g_{j+1}, x\right]}$ in $L / \gamma_{j+2}(L)$, we get:

$$
\overline{\left[g_{j}+g_{j+1}, x\right]}=\overline{\left[g_{j}, x\right]}+\overline{\left[g_{j+1}, x\right]}=\overline{0} .
$$

Hence $\overline{\left[g_{j}, x\right]} \in \gamma_{j+2}(L)$ and $g_{j} \in \gamma_{j+1}(L)$. But $g_{j}$ is a non-empty sum of independent left-normed basic commutators all of whose length is exactly $j$, so $g_{j}$ cannot also be a sum of left-normed basic commutators of length $\geq j+1$ (Lemma 3). Therefore $\left[g_{j}, x\right]=0$, which implies that $g_{j}$ commutes with all elements of the paragenerating set.

Now, since $L$ is parafree,

$$
L / \gamma_{n+1}(L) \cong F / \gamma_{n+1}(F), n=1,2,3, \ldots
$$

$F / \gamma_{n+1}(F)$ is free center-by-metabelian nilpotent, and its center is $\left(F^{\prime \prime}+\gamma_{n}(F)\right) / \gamma_{n+1}(F)$; thus the center of $L / \gamma_{n}(L)$ is $\left(L^{\prime \prime}+\gamma_{n}(L)\right) / \gamma_{n+1}(L)$. Since $g_{j}$ is a sum of left-normed basic commutators, none of which lies in $L^{\prime \prime}$, we get

$$
g_{j} \in \gamma_{n}(L), n=1,2,3, \ldots
$$

Let $n=j+1$ : then $g_{j} \in \gamma_{j+1}(L)$. But $g_{j} \notin \gamma_{j+1}(L)$. Therefore, $g_{n}=0$ for $n=2,3, \ldots$, and $Z(L)=L^{\prime \prime}$.

Lemma 5. If a Lie algebra $T$ is residually nilpotent, then the algebra $T / Z(T)$ is residually nilpotent.

Proof. Let $T$ be a residually nilpotent Lie algebra and $u \in \bigcap_{n=1}^{\infty} \gamma_{n}(T / Z(T))$. Then for all $n$

$$
u \in \gamma_{n}(T / Z(T))=\gamma_{n}(T)+Z(T) / Z(T) .
$$

Let $u=a+Z(T)$, where $a \in \gamma_{n}(T)+Z(T)$. It is clear that

$$
a \in \bigcap_{n=1}^{\infty} \gamma_{n}(T)+Z(T) .
$$

Since $T$ is residually nilpotent, then $a \in Z(T)$ and $u=Z(T)$. Hence $T / Z(T)$ is residually nilpotent.

Proof of Theorem 2: By Lemma 4, we get

$$
L / Z(L)=L / L^{\prime \prime}
$$

By Lemma $5, L / L^{\prime \prime}$ is residually nilpotent; hence we have 


$$
\Delta_{w}\left(L / L^{\prime \prime}\right)=\{0\} .
$$

Theorem 3. Let $P$ be a parafree Lie algebra. Then the algebra $\left(P / P^{\prime \prime}\right) / \Delta_{w}\left(P / P^{\prime \prime}\right)$ is a parafree metabelian Lie algebra.

Proof. For the proof, you may see [12].

Corollary 1. Let $L$ be parafree center-by-metabelian Lie algebra. Then $L / L^{\prime \prime}$ is parafree metabelian.

Proof. Let $L$ be a parafree Lie algebra. Then by the Theorem 3 , the algebra $\left(L / L^{\prime \prime}\right) / \Delta_{w}\left(L / L^{\prime \prime}\right)$ is a parafree metabelian Lie algebra. By hypothesis, $L$ is parafree centre-by-metabelian, so by Theorem 2, we have

$$
\Delta_{w}\left(L / L^{\prime \prime}\right)=0 .
$$

Therefore $L / L^{\prime \prime}$ is a parafree metabelian Lie algebra.

\section{4. acknowledgements}

The authors would like to thank Professor Naime Ekici for her supports and valuable comments and suggestions to improve the quality of the paper.

\section{References}

[1] Baumslag, G.: Groups with the Same Lower Central Sequence as a Relatively Free Group I. The Groups. Trans. Amer. Math. Soc., 129, 308-321 (1967).

[2] Baumslag, G.: Groups with the Same Lower Central Sequence as a Relatively Free Group. II Properties. Trans. Amer. Math. Soc. 142, 507-538 (1969).

[3] Baumslag, G.: Parafree Groups. Progress in Math. 248, 1-14 (2005).

[4] Baumslag, G., Cleary, S.: Parafree one-relator Groups. Journal of Group Theory. 9, 191-201 (2006).

[5] Baumslag, G., Cleary, S., Havas, G.: Experimenting with infinite group. Experimental Math. 13, 495-502 (2004).

[6] Baur, H.: Parafreie Lie Algebren und Homologie. Ph.D. thesis. Eidgenoessischen Technischen Hochschule Zuerich (1978).

[7] Baur, H.: A Note on Parafree Lie Algebras. Commun. in Algebra. 8(10), 953-960 (1980).

[8] Bokut, L.A., Kukin G. P.: Algorithmic and Combinatorial Algebra. Kluwer Academic Publishers. Dordrecht. The Netherlands (1994).

[9] Ekici, N., Velioğlu, Z.: Unions of Parafree Lie Algebras. Algebra. 2014, Article ID 385397, (2014).

[10] Ekici, N., Velioğlu, Z.: Direct Limit of Parafree Lie Algebras. Journal of Lie Theory. 25(2), 477-484 (2015).

[11] Velioğlu, Z.: Subalgebras and Quotient Algebras of Parafree Lie Algebras. IJPAM. 83, 507-517 (2013).

[12] Velioğlu, Z.: Parafree Metabelian Lie algebras which are Determined by Parafree Lie Algebras. Commun. Fac. Sci. Univ. Ank. Ser. A1. Math. Stat. 68(1), 883-888 (2019).

\section{Affiliations}

\section{ZEHRA VELIOĞLU}

ADDRESS: Harran University, Dept. of Mathematics, Sanliurfa, Turkey.

E-MAIL: zehrav@harran.edu.tr

ORCID ID:0000-0001-7151-8534 